NOTA CIENTÍFICA

\title{
Presencia y distribución de dos sub-especies de Eurema agave (Lepidoptera, Pieridae) en Costa Rica
}

\section{Presence and distribution of two sub-species of Eurema agave (Lepidoptera, Pieridae) in Costa Rica}

\author{
Jim Cordoba-Alfaro ${ }^{1}$ y Luis Ricardo Murillo-Hiller ${ }^{2}$
}

\begin{abstract}
Bachillerato en Biología, Universidad Nacional de Costa Rica. jim.cordoba@gmail.com

2 Centro de Acción Social e Investigación en Mariposas (CASIEM) Escuela de Biología, Universidad de Costa Rica.

murillohiller@gmail.com
\end{abstract}

Presentado: $\quad$ 12/02/2010

Aceptado: $\quad 23 / 07 / 2010$

Publicado online: 23/06/2011

\begin{abstract}
Resumen
Austin (1992) reportó a Eurema a. agave (Cramer 1775) para el Caribe de Costa Rica; sin embargo, en realidad lo que encontró fue a E. a. millerorum descrita por Bousquets \& Luis-Martinez (1987) para el Caribe mexicano. La presencia de Eurema a. agave es confirmada en este trabajo por especimenes recolectados en la vertiente Pacífica y Atlántica de Costa Rica. Además se detallan aspectos de la distribución de ambas subespecies.

Palabras clave: Distribución, Coliadinae, Eurema agave millerorum, alopatría, taxonomía.

\section{Abstract}

Austin (1992) reported Eurema a. agave (Cramer 1775) to the Caribbean of Costa Rica. However, he actually had found E. a. millerorum, described by Bousquets \& Luis-Martinez (1987) for the Caribbean of Mexico. The presence of Eurema a. agave is confirmed on this paper with information of specimens collected in the Pacific and Atlantic slopes of Costa Rica. Aspects on distribution of both subspecies are included.
\end{abstract}

Keywords: Distribution, Coliadinae, Eurema agave millerorum, allopatry, taxonomy.
Las mariposas el género Eurema (Hübner, 1819) (Pieridae) se caracterizan por ser de tamaño pequeńo (alrededor de 30 $\mathrm{mm}$ de envergadura), de color amarillo, anaranjado o blanco con los márgenes negros en las alas anteriores. Sus especies son abundantes particularmente en zonas de vegetación secundaria o alterada por las actividades humanas (DeVries 1987). Este género se distribuye tanto en el viejo mundo como en América. Desde Estados Unidos hasta Chile se conocen 19 especies (Lamas 2004), de las cuales en Costa Rica han sido reportadas siete desde el nivel del mar hasta los 2000 en las vertientes pacifica y atlántica (DeVries 1987).

El primer listado de especies de Eurema para Costa Rica fue realizado por DeVries (1983); posteriormente en su libro de las mariposas de Costa Rica (1987) considera que al menos dos especies son de poblaciones locales o reducidas. El 24 de julio de 1987, Ángel Solís recolectó el primer espécimen de E. agave (Cramer 1775) para Costa Rica en la localidad de Fila Esquinas de Puntarenas (200 m sobre el nivel del mar) en la vertiente Pacífica, y que se encuentra depositado en el Museo Nacional de Costa Rica (MNCR), bajo el nombre de Eurema daira eugenia (Wallengren1860), número de catalogo A00-004474.

Sin embargo, la presencia de $E$. agave en Costa Rica solo necesitaba ser probada con especimenes testigo, ya que desde Godman \& Salvin (1889-1890) se reportan recolectas en Chontales de Nicaragua y un macho ilustrado en sus láminas procedente de Panamá. Los próximos registros $E$. agave para Costa Rica los publicó Austin (1992) a partir de un macho recolectado en Puerto Viejo de Heredia y dos hembras de Puerto Viejo de Limón, ambas localidades entre los 0 y los $150 \mathrm{~m}$ de altitud en la vertiente Caribe.

Finalmente, los últimos especímenes recolectados de $E$. agave son una hembra procedente de playa Esterillos Este $\left(9^{\circ} 31^{\prime} 25,07^{\prime \prime} \mathrm{N}, 84^{\circ} 27^{\prime} 04,71^{\prime \prime W}\right)$ en Puntarenas, al nivel del mar, el 4 de enero de 2009 y un macho capturado en Río Verde de Pococí en Limón (10²' $24,34^{\prime \prime} \mathrm{N}, 83^{\circ}$ 46'19,62"W), a 278 $\mathrm{m}$ de altitud, el 22 de noviembre de 2010, ambos especímenes recolectados por JC-A y depositados en la colección personal Córdoba-Alfaro (CPCA), bajo los números de catalogo $30 \mathrm{MH}$ NJC y 270MHNJC respectivamente (Fig. 1).

Al comparar la figura 4 de Austin (1992), con los individuos recolectados en la vertiente del Pacífico de Costa Rica (por A. Solís), se observa que las alas posteriores son inmaculadas dorsalmente, pues no presenta un borde obscuro (Lorente-Boustquets \& Luis-Martinez 1987), a diferencia de los individuos del Pací-

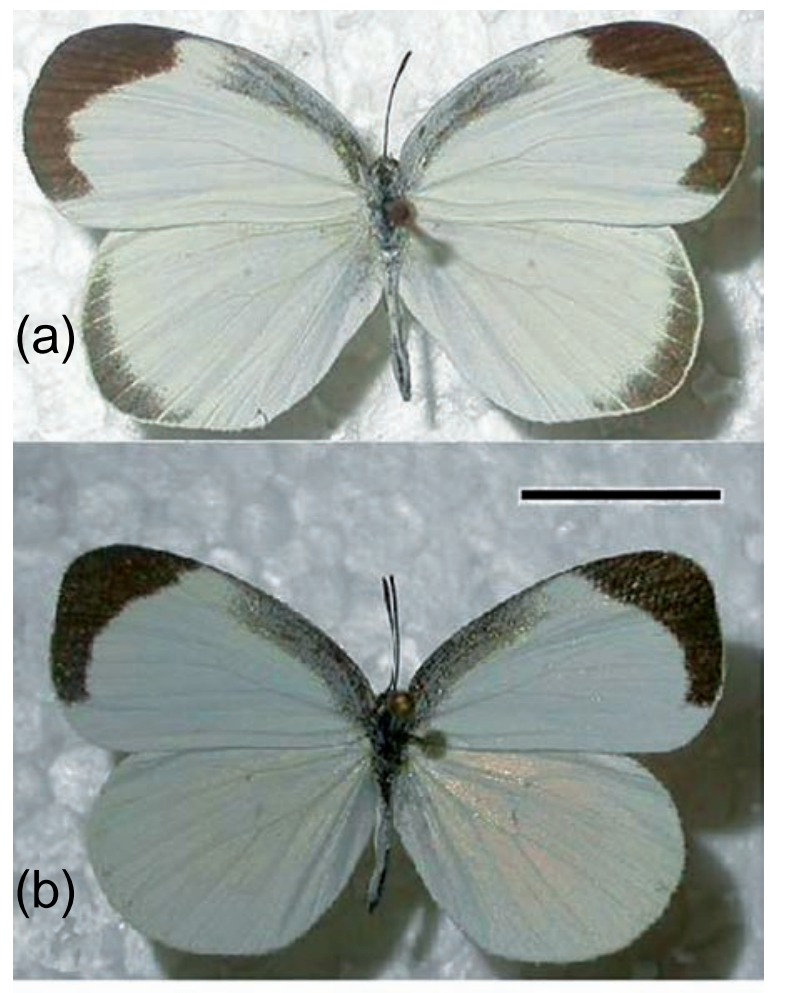

Figura1. (a) Eurema a. agave (Boisduval): $q$ - COSTA RICA: Playa Esterilos Este $\left(9^{\circ} 31^{\prime} 25,07^{\prime \prime} \mathrm{N}, 84^{\circ} 27^{\prime} 04,71^{\prime \prime} \mathrm{W}\right)$ Puntarenas. (b). Eurema a. millerorum Boustquets \& Luis-Martinez : ${ }^{\lambda}$ COSTA RICA: Rio Verde (10¹2'24,34"N, 8346'19,62”W), Pococí, Limón. 
fico. Al comparar los especímenes del Pacifico con los ilustrado en Godman \& Salvin (1889-1890) se observa que los fenotipos en las alas posteriores son idénticos. En esta última ilustración, el espécimen está identificado como Terias mana, hoy considerada por Lamas (2004) como E. a. agave.

Eurema agave millerorum Boustquets \& Luis-Martinez 1987 fue descrita como nueva sub-especie en Tabasco, en el Caribe mexicano y los autores señalan que posiblemente esta sub-especie alcance áreas del norte de Centroamérica ya que hacia Nicaragua, Costa Rica y Panamá lo que se hallaría sería E. agave mana, considerada en realidad $E$. agave agave. Al analizar la figura 4 de Austin (1992), se evidencia que la hembra ilustrada es en realidad la $E$. agave millerorum que se describió en México, y que posiblemente alcance no solo el Caribe de Costa Rica sino hasta el Caribe panameño. Mientras que, los especimenes recolectados en el Pacífico costarricense son $E$. agave agave.

En conclusión E. agave millerorum estaría restringida a las tierras bajas de la vertiente Caribe desde México hasta Costa Rica y E. agave agave se reporta por primera vez para Costa Rica para las vertientes Caribe y Pacífica.

Material Examinado: 38 especímenes (13우요

Costa Rica, Puntarenas, Fila espinas, 200msnm, (1 9 ) 24/7/1987, A. Solís. (MNCR). Costa Rica, Puntarenas, Playa Esterillos Este, $\left(9^{\circ} 31^{\prime} 25,07^{\prime \prime N}, 8^{\circ} 27^{\prime} 04,71^{\prime \prime W) ~} 0\right.$ msnm, 4/I/2009, J. Córdoba (19). (CPCA). Costa Rica, Limón, Pococí, Guápiles, Río Verde (10²12'24,34"N, 8346'19,62"W) 278msnm, (1ठ), 22/XI/2010, J. Córdoba, (CPCA); (7우,
14ல̂), 22/I/2011, J. Córdoba \& I. Rodríguez, (CPCA). Costa Rica, Limón, Pococí, Guápiles, Río Verde $\left(10^{\circ} 12^{\prime} 24,34 " \mathrm{~N}\right.$, 8346'19,62"W) 278 msnm, (6今̄, 1우), 22/I/2010, J. Córdoba, (MNCR). Perú, Iquitos, Loreto, $100 \mathrm{msnm},(3+$, 4ठ), 12/ XII/2010, R. Westerduijn, (CPCA).

\section{Agradecimientos}

Agradecemos el apoyo brindado por el Museo Nacional de Costa Rica, en especial a Germán Vega y Cecilia Pineda, al Museo de Insectos de la Universidad de Costa Rica por prestar sus instalaciones, a Rob Westerduijn de la Universidad de Wageningen por la donación de muestras recolectadas en Perú.

\section{Literatura citada}

Austin G. T. 1992. New and additional records of Costa Rican Butterflies. Tropical Lepidoptera, 3(1): 25-33.

DeVries P. J. 1983. Checklist of butterflies, p. 654-678. In: D. Janzen (ed.). Costa Rican Natural History. The Universtity of Chicago Press. Chicago. U.S.A.

DeVries P. J. 1987. The Butterflies of Costa Rica and Their Natural History: Papilionidae, Pieridae, Nymphalidae. Princeton University Press: New Jersey. 327 pp.

Godman F.D. \& O. Salvin.1889-1890. Biologia Centrali-Americana. Zoologia. Insecta. Lepidoptera. Rhopalocera. Vol I. (texto y III laminas).

Lamas G. 2004. Pieridae, p. 99-117. In: G. Lamas (ed.). Atlas of the neotropical Lepidoptera: Part 4A Hesperioidea-Papilionoidea. Scientific Publishers. Florida. 439 pp.

Lorente-Boustquets J. \& A. Luis-Martinez. 1987. Una nueva subespecie de Eurema agave Cramer (Lepidoptera: Pieridae; Coliadinae). Folia Entomológica Mexicana 71: 17-25. 